\title{
Analysis of Student Questions in MOOC Physics Lectures: The Case of the Khan Academy
}

\author{
Sohee JEONG · Jinwoong SONG* \\ Department of Physics Education, Seoul National University, Seoul 08826, Korea \\ Wonyong PARK \\ Department of Education, University of Oxford, Oxford OX2 6PY, United Kingdom
}

(Received 20 February 2019 : revised 1 April 2019 : accepted 4 April 2019)

\begin{abstract}
This study reports an analysis of 5,538 questions, asked by learners after studying the classical physics and the quantum physics units in Khan Academy's physics course, a massive open online course (MOOC) platform popular worldwide. Based on Etkina's and Harper et al.'s categories, the questions were coded into four levels of difficulty and six topics. In addition, the questions were further analyzed using semantic network analyses. In terms of the level of difficulty, classical physics showed similar numbers of high- and medium-level questions while in quantum physics, the number of high-level questions was significantly higher. In terms of the topic of the question, conceptual questions were the most frequently raised questions both in classical physics and quantum physics. In classical physics, questions about "concept" and "knowing" were frequently raised while the frequency of "concept" questions was significantly higher in quantum physics. The massive questions recorded on the MOOC platform allowed for effective analyses of physics questions by students. Educational implications and a prospective research agenda are proposed based on the findings.
\end{abstract}

PACS numbers: 01.40.-d, 01.50.H-, 01.50.ht

Keywords: MOOC, Classical physics education, Quantum physics education, Question level, Question topic

\section{MOOC 물리학 강의의 학생 질문 분석: 칸 아카데미를 중심으로}

\author{
정소희 · 송진웅*
}

서울대학교 물리교육과, 서울 08826 , 대한민국

\section{박원용}

Department of Education, University of Oxford, Oxford OX2 6PY, United Kingdom

(2019년 2월 20일 받음, 2019년 4월 1일 수정본 받음, 2019년 4월 4일 게재 확정)

본 연구는 대규모 온라인 공개 수업 (MOOC) 플랫폼 중 수학, 과학 분야에서 대표적인 칸 아카데미 (Khan Academy) 물리학 강의 중 고전물리학과 양자물리학을 학습한 학습자가 댓글로 단 질문 5,538 개를 분석하였다. Etkina의 4 가지 질문 수준 및 Harper et al.의 6 가지 질문 주제 범주를 기준으로 질문을 분류한 후, 언어 네트워크 분석을 실시하여 질문의 내용을 심층적으로 분석하였다. 질문의 수준 면에서 고전물리학에서는 중간 수준과 높은 수준의 질문이 비슷한 정도로 높게 나타났으며, 양자물리학에서는 
높은 수준의 질문이 현저하게 많이 나타났다. 질문의 주제 측면에서는 고전물리학과 양자물리학 모두 개념에 관한 질문이 가장 많았다. 고전물리학에서 개념 질문과 지식 질문이 비슷하게 많이 나타난 반면, 양 자물리학에서는 개념에 관한 질문의 비율이 현저하게 높고 나머지는 미미하였다. $\mathrm{MOOC}$ 플랫폼에 축적된 방대한 댓글 질문 자료를 토대로 전통적인 교실 상황에서의 질문 연구에 비해 다양한 유형의 물리학 질문을 효과적으로 분석할 수 있었다. 연구 결과를 바탕으로 교육적 시사점 및 후속 연구 과제를 제안하였다.

PACS numbers: 01.40.-d, 01.50.H-, 01.50.ht

Keywords: MOOC, 고전물리학, 양자물리학, 질문 수준, 질문 주제

\section{I. 서론}

2013년 공개된 미국 차세대 과학교육표준(Next Generation Science Standards) 은 모든 학생들에게 공평한 학습 의 기회를 주기 위하여 유치원부터 고등학교까지 배워야 할 과학교육의 내용과 체재를 마련함으로써 과학교수학습의 미래상을 제시하였다. 이에 따라 교육 지원 체제에서의 변 화가 있었으며, 특히 네트워크 간접 자원의 활용과 디지털 자원의 이용 등의 내용이 표준안에 새롭게 포함되었다 [1]. 이와 관련하여 현재 디지털 자원의 이용 중 대표적인 형태로 대규모 온라인 공개 수업 (Massive Open Online Course, $\mathrm{MOOC})$ 가 각광받고 있으며, 그 필요성이 중시되고 있다. $\mathrm{MOOC}$ 는 개인의 학습량과 숙달도에 맞추어 학습 속도와 내용을 조절하려는 학습자들과 시공간에 제한받지 않고 학습하려는 학습자들이 늘어나면서 학생중심 교육매체의 중요 사례로 부각되고 있다 [2].

$\mathrm{MOOC}$ 는 전세계 모든 사람들을 대상으로 제공하는 온 라인 공개 수업으로서 연령과 소속 등의 제한 없이 누구나 무료로 수강할 수 있는 강의이다 [3]. MOOC를 이용한 학 습은 학습자가 언제, 어떻게, 어떤 콘텐츠를 가지고 학습할 지를 스스로 선택하고 결정할 수 있다는 점에서 큰 의의를 가진다 [2]. 초기의 $\mathrm{MOOC}$ 는 교수자가 일방적으로 강의하 는 동영상 형태로 시작되었다. 하지만 현재는 교육자료와 학습공동체에 대한 열린 접근 및 집합적인 노력을 지속한 결과, 다수의 학습자들이 소외되지 않고 자신의 의견을 개 진하여 수업에 생산자로 참여하는 구조로 변화하였다. 이와 더불어 동료 학습자와 토론하고 협력하며 교사에게 실시간 으로 질문을 하고 이에 대한 즉각적인 답변이 이루어지는 등 쌍방향 소통으로 교육형태가 변화하고 있다 [2].

이러한 형태의 $\mathrm{MOOC}$ 중 하나로 칸 아카데미(Khan Academy, www.khanacademy.com) 가 있다. 칸 아카데미 는 표준 교실 모델에서 벗어나는 형태를 지향한다 [4]. 2006 년에 설립된 비영리 단체인 칸 아카데미는 세계적인 수준 의 교육을 제공하는 가장 널리 알려진 무료 온라인 교육 플랫폼 중 하나이다. 2009년 초반을 기준으로 수만 명의

*E-mail: jwsong@snu.ac.kr
학습자들이 칸 아카데미를 이용하기 시작하였고, 그해 8 월에는 빌 게이츠가 설립한 게이츠 재단(Bill \& Melinda Gates Foundation) 의 투자지원을 받기에 이르렀다 [4]. 칸 아카데미가 다른 온라인 $\mathrm{MOOC}$ 플랫폼과 차별화되는 점 은 단순히 온라인상에서 교육을 제공하는 것에 그치지 않 고 교실수업에서의 칸 아카데미 컨텐츠 활용을 적극적으로 지원하고 있다는 점이다. 국내에서도 한국어 칸 아카데미 사이트 [5]를 개설하는 한편 교사를 대상으로 칸아카데미 활용 방안 온라인·오프라인 연수를 꾸준히 진행하고 있다 [6]. 특히 칸 아카데미는 물리 및 수학 분야에 특화된 개방형 온라인 학습도구로 각광받고 있다. 이처럼 방대한 규모 및 현지화를 통한 국내 영향력의 확대를 고려할 때, 칸 아카데 미는 물리교육 분야의 대표적인 $\mathrm{MOOC}$ 활성화 사례로서 주목할 필요가 있다.

특히 칸 아카데미는 학습자의 적극적인 참여와 자기주도 적 학습을 유도하기 위해 학습자들이 수강한 모든 강의의 질문란에 댓글로 질문할 수 있는 시스템을 갖추고 있다. 이 러한 질문들은 학습 맥락에 맞게 학습자들이 댓글로 작성할 수 있으며, 학습자 스스로가 당면한 문제를 해결하기 위한 적극적인 자세를 취할 수 있게 하는 하나의 좋은 수단이 된 다. 또한 이렇게 댓글로 제시된 질문들에 학습자들이 투표 기능을 통해 동의를 표현할 수 있도록 하였다. 이는 여러 학습자들이 궁금해하는 질문이 무엇인지 잘 알 수 있도록 해 주며, 댓글 질문에 자신이 생각한 답변 및 또다른 질문을 덧붙임으로써 다른 학습자들과 의사소통할 수 있는 기능을 가진다.

본 연구는 칸 아카데미 물리학 강의 수강생들이 온라인 상에 댓글 형식으로 제시한 질문을 질문의 수준과 주제 면에 서 분석하였다. 고전물리학 단원에서 구심력과 중력 단원은 고전역학 중 뉴턴 역학의 개념적 이해를 심화시키는 좋은 학습 주제가 되지만, 개념의 부주의한 해석이 자주 발생되어 오개념이 유발되는 단원이기도 하다 [7]. 또한 고전물리학 에서 중요하게 다루어지고 있는 법칙들 중 하나인 뉴턴의 운동 법칙에서 중력과 관련 있는 부분을 자세히 다루고 있 어, 고전물리학의 대표적 단원으로 선정하였다. 구심력 및 중력은 고전물리학의 기본이 되는 힘, 속도, 가속도 등의 물리량뿐만 아니라 뉴턴의 운동 3 법칙이 모두 관여하는 단 
원으로서 내용상 종합적인 성격을 가지며 이에 따른 이해의 어려움이 자주 보고되어 왔다 [8]. 또한 본 연구의 목적 및 연구방법의 관점에서 방대한 양의 고전물리학 단원 질문 전체를 분석하는 것보다 양자물리학 단원의 질문의 수와 양적으로 비슷하면서도 앞서 언급한 고전물리학의 다양한 어려움들을 고루 반영할 수 있다는 점에서 고전물리학 영역 중 '구심력과 중력' 단원을 선택하였다.

한편 양자물리학은 미시적인 세계를 다루며, 내용이 추상 적이고 직관적으로 받아들이기 어렵다는 특징 때문에 학습 자들이 개념이해에 있어서 많은 어려움을 갖는 내용이기도 하다. 양자물리학의 여러 개념 중 불확정성 원리에 관한 연구들을 살펴보면, 학습자들은 '오류, 편차' 라는 개념을 불확정성으로 표현하고 있으며, 이를 고전물리학적 내용과 구분하지 못한다는 것을 확인할 수 있다 [9]. 또한 학습자 들은 위에서 언급한 불확정성의 원리 외에 입자-파동 이중 성과 터널링 효과에 관해서도 어려움을 갖고 있다. 입자파동성도 개념적으로 한가지 개념으로 설명하기 어려우며, 두개 이상의 연관된 개념을 통해 현상을 이해할 수 있다 [10]. 또한 학습자들이 이러한 개념 및 실험에 대한 이해가 부족한 이유는 실험의 정확성, 측정 등과 같은 실험과 관련 된 항목이 개인의 경험 부족 때문이라는 연구가 있다 [11]. 이와 더불어 학생들이 양자물리학을 학습하는 데 있어서 자각하는 어려움으로 수학적인 어려움을 들 수 있다 [12]. 양자물리학에서 불확정성 원리에 대한 선행 연구들도 개념 적인 측면과 수식적 측면으로 나누어 분석하며, 관계식의 적용을 통한 문제 풀이와 개념의 의미에 대한 이해에 있어 서 학습자들이 겪는 어려움이 크다는 것을 지적하였다 [9]. 양자물리학 단원의 어려움의 원인은 위의 선행 연구들에서 살펴본 것과 같이 개념적인 내용과 수학적인 내용의 어려움 으로 나눌 수 있으며, 이 두 가지 영역의 어려움이 학습자의 질문에 반영되어 나타날 것으로 기대할 수 있다.

본 연구의 목적은 $\mathrm{MOOC}$ 기반 물리학 학습환경에서 대 조적인 성격을 지닌 두 단원, 즉 고전물리학 중 구심력과 중력 단원의 질문과 양자물리학 단원에서 제기되는 질문을 조사함으로써 이 두 가지 하위 내용영역에서 나타나는 학생 질문의 양상과 특징을 비교, 대조하는 것이. 연구 문제는 다음과 같다.

1. 칸 아카데미 물리학 강의의 고전물리학 단원과 양자 물리학 단원에서 학생들이 제기하는 질문의 수준은 어떠한가?

2. 칸 아카데미 물리학 강의의 고전물리학 단원과 양자 물리학 단원에서 학생들이 제기하는 질문의 주제는 어떠한가?

\section{II. 물리학 교수·학습에서 질문의 역할}

교수학습의 과정에서 학습자들이 제기하는 질문은 학습 에서 중요한 역할을 하고 있으며, 질문의 중요성 또한 교육학 자들 사이에서 오랫동안 강조되어 왔다 [13]. 질문을 한다는 것은 학습자가 학습한 것을 얼마나 이해했는지 확인하는 데 도움을 주고, 학습 전략을 재정비하는 데 필요한 피드백의 원천이 된다 [14]. 질문하기 (questioning) 는 비판적 사고, 창의적 사고, 문제 해결 과정을 구조화하는 과정 중의 하나 이다 [15]. 또한 질문하기를 통한 교수법은 교사의 역할에도 많은 영향을 미친다. 스탠퍼드 대학에서 개발한 Stanford Mobile Inquiry-based Learning Environment (SMILE)을 기반으로 한 연구는 교실에서 ICT와 질문 교육, 모바일 애 플리케이션의 결합을 통해 교사-학생 관계를 변화시키고, 학습곡선을 가속화하는 등의 교육효과를 창출할 수 있으며, 이에 따라 교실에서 전형적인 학생과 선생님의 역할을 변 화시킬 수 있다고 보고한 바 있다 [16].

학습에서 질문에 대한 관심이 늘어나고 중요성이 강조 되면서 질문하기에 대한 연구가 활발히 이루어지고 있다. 질문 연구가 갖는 가치는 학습 환경이나 학습 주제에 따라 조금씩 달라지는데, 물리학과 같이 자연과학적, 논리적 색 채가 강한 과목의 경우 그 특성상 학습자가 가지는 질문이 학습자의 학습 상태를 나타내는 지표가 될 수 있다 [17]. 따 라서 물리학을 학습한 학생들의 질문을 통해서 교사는 학 습자의 학습에 대한 이해 정도와 수준을 파악할 수 있으며, 학생들이 이해한 것과 이해하지 못한 것이 무엇인지에 대한 정보를 얻을 수 있다 [18].

학습자가 과학적 개념을 이해하는 과정에 맞추어 학생 질문의 주제를 파악하는 것은 교사와 학생에게 많은 영향을 준다. 우선, 질문의 주제는 학생의 과학에 대한 흥미와 연 결된다. 학생의 과학적 흥미는 학생들의 질문을 유발하는 동기가 되며, 이것은 점차 높은 수준의 과학적 주제로 확 장되어 학습자 스스로 연구 질문을 이끌어 낼 수 있다 [15]. 이를 통하여 교사는 학습자를 위한 학습 내용을 체계적으로 구성하여 제시할 수 있으며, 이러한 경험이 있는 교사가 질 문에 대해 모델링해 주거나 연습을 시킨 학생은 그렇지 않 은 집단보다 더 잠재적인 질문을 제시하였다는 보고도 있다 [19]. 또한 교사는 학습자가 제기한 질문의 주제를 파악하여 이를 바탕으로 학습적 상호작용을 유도할 수 있으며, 학습한 지식과의 개념적인 확장을 이끌어 낼 수 있다 [20]. 질문의 주제 연구는 학습자의 오개념 연구로 연구 대상을 확장할 수 있어, 학생들의 물리학 오개념을 교정하기 위한 적절한 교수전략으로 발달시키는 데 도움이 된다 [21]. 요컨대 물 리학 학습자가 가지는 질문은 학습자가 경험하는 어려움의 정도와 과학적 개념 및 주제에 대한 이해를 반영하는 것으 
로 볼 수 있으며, 교사 또는 교수매체는 학생 질문을 적절히 활용함으로써 물리학 학습의 효과를 증진시킬 수 있다고 볼 수 있다. 교사가 이러한 질문을 통한 피드백을 바르게 이해한다면, 학생들이 생각하는 것과 필요한 것이 무엇인지 명확하게 알 수 있어 학생들이 처한 학습 상황을 분석하여 학습자에게 적절하고 유용한 수업 내용과 과제 등을 제공할 수 있다.

질문이 가지는 의미와 중요성은 과학교육과정에서도 꾸 준히 강조되고 있다. 과학적 흥미에 의해 생성된 학습자의 자발적인 질문은 과학 학습 과정에 대한 자연스러운 접근을 촉진한다는 보고가 대표적이다 [23]. 학생들의 자발적인 질문 활동을 중심으로 한 과학 수업에서는 학생들이 정답을 작성해야 한다는 강박 관념이 없기 때문에, 학생들의 사고를 촉진하고, 과학 개념 이해도 증진에 긍정적인 효과를 준다는 연구도 있다 [24]. 한편 이상적인 과학 수업에서는 질문하기 와 대답하기가 상호적으로 이루어져야 하지만, 일반적으로 질문하기가 대답하기보다 더 강조되고 있으며 [25], 학생이 제기하는 질문들은 때로는 연구가설 (research hypothesis) 과 같은 유용한 과학적 연구 질문으로 발전하기도 한다 [13].

상기한 여러 연구에서 언급한 과학교육에서 질문의 필 요성은 $\mathrm{MOOC}$ 기반 물리학 수업의 특징과 많은 연관성을 가진다. 따라서 $\mathrm{MOOC}$ 를 통한 물리학 수업에서 질문 분 석을 바탕으로 향후 온라인 기반 물리학 강의에서 학생의 질문에 기초한 유용한 피드백을 제공하기 위한 기초 정보를 얻을 수 있을 뿐만 아니라 물리학 교수학습에 대한 중요한 함의를 도출할 수 있을 것으로 기대된다. 종전의 $\mathrm{MOOC}$ 연구들은 대부분 특정 교과목과 주제에 직접적으로 연관 된 내용보다는 온라인 학습의 현황과 특징, 특정한 온라인 도구의 교수법적 효과 등 교과일반적인 (subject-general) 내용을 주로 다루어 왔다. 이러한 이유 때문에 특정 과목의 학습자들이 개방형 온라인 시스템에서 학습을 하면서 갖는 학습 단원별 어려움에 관한 연구는 드물다.

\section{III. 연구 방법}

\section{1. 연구 대상 및 자료 수집}

본 연구자는 칸 아카데미의 과학 강의 중 '물리' 에 있는 강의를 중심으로 자료를 수집하였다. 칸 아카데미의 물리학 강의에 포함된 19 개 단원의 431 차시 수업은 운동과 역학 단원의 내용을 좀더 세부적으로 8 개의 영역으로 나누었으 며, 유체, 열역학, 전자기학 등으로 전체 고전물리학 단원 을 구성하였다. 그리고 양자물리학 단원은 단일 영역으로 25 개의 강의로 구성하였다. 연구의 목적에 맞게 고전물리
학 단원 중 대표적인 '구심력과 중력 (centripetal force and gravitation)' 단원과 '양자물리학 (quantum physics)' 단원 을 선택하고, 두 단원을 수강한 학습자들이 강의 페이지에 들어가 질문란에 자유롭게 기술한 질문들을 수집하였다.

‘구심력과 중력' 단원은 고전역학 중 뉴턴 역학의 개념적 이해를 심화시키는 좋은 학습 주제이지만, 개념의 부주의한 해석이 자주 발생되어 오개념이 유발되는 단원이기도 하다 [26]. 또한 고전물리학에서 중요하게 다루어 지고 있는 법칙 들 중 하나인 뉴턴의 운동법칙에서 중력과 관련 있는 부분 을 자세히 다루고 있으며, 힘, 속도, 가속도, 원운동 등 고전 물리학의 중요 개념들에 대한 종합적인 이해를 요구한다는 점에서 고전물리학의 대표 단원으로 선정하였다. 이와 함께 해당 단원에서 제기된 학생 질문의 수가 양자물리학 단원 질문의 수와 유사하다는 점도 대표 단원의 선정 과정에서 고려되었다. 칸 아카데미에 게시된 구심력과 중력 단원의 강의는 크게 3 개 소단원, 즉 원운동과 구심가속도 (circular motion \& centripetal acceleration), 구심력 (centripetal forces), 뉴턴의 중력 법칙(newton's law of gravitation)으 로 나누어져 있으며, 그 구성은 Table 1 과 같다. 각 강의는 15 분 내외로 짧게 이루어져 있으며, 예시 문항의 풀이는 개 념 설명과는 별개의 강의로 나누어 제시하였다. 양자물리 학 단원의 강의는 광자(photons), 원자와 전자 (atoms and electrons), 양자수와 궤도 (quantum numbers and orbits), 핵 (nuclei)으로 나뉘며, 세부적인 강의의 내용은 Table 2 와 같다. 양자물리학 강의들 중 질문 수는 방사선 핵의 붕괴형 식에 관한 질문이 가장 많았으며, 강의시간은 핵의 붕괴에 관한 강의가 가장 길었다.

이같은 기준에 따라 구심력과 중력 단원 2,753 건, 양자물 리학 단원 2,785 건으로 총 5,538 건의 질문을 수집하였다. 단, 사용자의 댓글에 달린 답글은 질문의 형식을 취하지 않거나 내용상 중복되는 경우가 많아 수집 대상에 포함하지 않았다.

\section{2. 분석 도구 및 절차}

칸 아카데미 물리학 질문 분석을 위해 Etkina 의 질문 수 준 분류와 (Table 1) [18] 학습자의 질문의 주제를 분류하기 위해 Harper et al.의 질문 주제 분류를 사용하였다 (Table 2) [28]. 질문 수준과 주제 각각을 1 차적으로 분류하기 위 해 2 인의 연구자가 200 개의 질문을 임의로 추출하여 예비 분석하면서 협의를 통해 각 범주에 해당하는 핵심어를 선 정하였으며, 분석에 대한 세부 기준을 마련하였다. 이처럼 핵심어를 사용한 텍스트 자료 분석은 과학교육 분야에서 자주 사용되는 연구방법이나 [29], 질문의 맥락을 고려하여 
Table 1. Organization of the centripetal force and gravity unit in Khan Academy.

\begin{tabular}{|c|c|c|}
\hline Unit & Number of questions & Duration (m:s) \\
\hline \multicolumn{3}{|l|}{ Part 1. Circular motion \& Centripetal acceleration } \\
\hline Race cars with constant speed around curve & 97 & $4: 48$ \\
\hline Centripetal force and acceleration intuition & 239 & $10: 46$ \\
\hline Visual understanding of centripetal acceleration formula & 144 & $9: 47$ \\
\hline Optimal turns at Indianapolis Motor Speedway with JR Hildebrand & 30 & $6: 24$ \\
\hline Calculus proof of centripetal acceleration formula & 112 & $14: 21$ \\
\hline Loop de loop question & 16 & $3: 00$ \\
\hline Loop de loop answer part 1 & 50 & 6:04 \\
\hline Loop de loop answer part 2 & 67 & $4: 47$ \\
\hline \multicolumn{3}{|l|}{ Part 2. Centripetal Forces } \\
\hline Centripetal force problem solving & 67 & $14: 59$ \\
\hline Yo-yo in vertical circle example & 53 & $10: 30$ \\
\hline Bowling ball in vertical loop & 43 & $8: 48$ \\
\hline Mass swinging in a horizontal circle & 33 & $12: 38$ \\
\hline \multicolumn{3}{|l|}{ Part 3. Newton's law of Gravitation } \\
\hline Introduction to gravity & 665 & $15: 42$ \\
\hline Mass and weight clarification & 262 & $10: 40$ \\
\hline Gravity for astronauts in orbit & 213 & $7: 11$ \\
\hline Would a brick or feather fall faster? & 181 & $10: 34$ \\
\hline Acceleration due to gravity at the space station & 95 & $10: 12$ \\
\hline Space station speed in orbit & 98 & $6: 35$ \\
\hline Introduction to Newton's law of gravitation & 139 & $8: 37$ \\
\hline Gravitation & 149 & $8: 16$ \\
\hline Total & 2,753 & 3:03:39 \\
\hline
\end{tabular}

분석할 필요가 있기 때문에 연구자의 추가적인 판단이 반드 시 필요하다. 핵심어의 부재, 복수 핵심어 존재 및 기타 예외 적인 상황에 대한 판단 기준을 합의한 이후 1 인의 연구자가 전체 질문을 분석하였다. 이후 각 단원별로 가장 빈도가 높은 수준의 질문에 대한 심층적 분석을 위해 추가적으로 언어 네트워크 분석을 실시하였으며, 그 방법 및 절차는 해당 항목에 별도로 기술하였다.

\section{1) 질문 수준 분석}

Etkina의 질문 수준 분류 틀 (Table 1)은 물리학 학습자의 질문을 통해 학습의 이해정도를 파악하기 위해 제안되었다 [18]. Table 1은 Etkina에 의해 정의된 4 가지 질문 수준과 각 수준에 대한 설명, 그리고 질문 수준을 분류하기 위한 핵 심어(keywords) 를 나타낸 것이다. 핵심어는 본 연구에서 새롭게 추가된 것으로, 예를 들어 "introduce, define, what is, letter, unit, called, symbol” 등의 단어를 포함한 질문
들은 최소(minimal) 수준의 정의에 부합하는 바 이 범주로 1 차적으로 분류된 이후 연구자에 의한 2 차 분류로 넘겨졌 다. 핵심어의 선정 및 분류에 관한 상세한 절차는 논문의 연구방법 절에 기술하였다.

최소(minimal) 수준은 학생이 학습한 내용 중 기본적인 정의적 문제에 대한 질문이다. 학습자가 사실적인 정보에 대해 제기한 질문을 최소 수준으로 정의하였다 [18]. 학습 자가 학습 내용에 있어서 가장 기본적인 학습 목표를 잘 이 해하지 못했을 경우, 또는 개념을 제대로 이해하지 못했을 경우 학습자가 가지는 질문이 주로 이 범주에 속한다 [13]. 낮은 (low) 수준은 교사가 가르치는 데 있어서 사용하는 표 상 (그래프, 표, 사진, 언어적 표현 등) 들을 학습자가 이해하 는 데 어려움을 가지고 질문한 경우에 해당한다. 또한 학습 정보들을 단순히 비교하거나 차이점에 대해 의문을 가지는 경우도 학습의 표상을 이해하는 데 어려움을 가지는 것에 속하므로, 낮은 수준으로 분류하였다. 중간 수준 (medium) 의 질문은 학습자가 학습대상을 이해하는 데 있어 과정적인 어려움을 가진 경우로 정의된다. 최소 및 낮은 수준과는 달 
Table 2. Organization of the quantum physics unit in Khan Academy.

\begin{tabular}{|c|c|c|}
\hline Unit & Number of questions & Duration (m:s) \\
\hline \multicolumn{3}{|l|}{ Part 1. Photons } \\
\hline Photon Energy & 107 & $9: 17$ \\
\hline Photon Momentum & 18 & $2: 54$ \\
\hline Photoelectric effect & 254 & $10: 23$ \\
\hline \multicolumn{3}{|l|}{ Part 2. Atoms and Electrons } \\
\hline De Broglie wavelength & 55 & $11: 19$ \\
\hline Quantum Wavefunction & 55 & $10: 10$ \\
\hline Atomic Energy Levels & 88 & $9: 58$ \\
\hline Bohr model radii (derivation using physics) & 88 & $10: 51$ \\
\hline Bohr model radii & 30 & $6: 56$ \\
\hline Bohr model energy levels (derivation using physics) & 41 & 12:07 \\
\hline Bohr model energy levels & 114 & $9: 46$ \\
\hline \multicolumn{3}{|l|}{ Part 3. Quantum numbers and Orbits } \\
\hline Absorption and emission & 95 & $10: 29$ \\
\hline Emission spectrum of hydrogen & 169 & $10: 49$ \\
\hline Heisenberg uncertainty principle & 138 & $10: 18$ \\
\hline \multicolumn{3}{|l|}{ Part 4. Nuclei } \\
\hline Quantum numbers & 267 & $11: 59$ \\
\hline Quantum numbers for the first four shells & 102 & $12: 36$ \\
\hline Mass defect and binding energy & 62 & $11: 28$ \\
\hline Nuclear stability and nuclear equations & 82 & $8: 25$ \\
\hline Types of decay & 432 & $17: 02$ \\
\hline Writing nuclear equations for alpha, beta, and gamma decay & 50 & 8:06 \\
\hline Half-life and carbon dating & 265 & $12: 30$ \\
\hline Half-life plot & 28 & $6: 46$ \\
\hline Exponential decay formula proof (can skip, involves calculus) & 76 & $12: 21$ \\
\hline Introduction to exponential decay & 99 & $9: 18$ \\
\hline More exponential decay example & 62 & $7: 21$ \\
\hline Exponential decay and semi-log plots & 8 & $10: 15$ \\
\hline Total & 2,785 & $4: 13: 24$ \\
\hline
\end{tabular}

리 단순히 정보에 대해 물어보는 것이 아니라, 이해하면서 학습자 스스로 개념을 재구성하는 질문들이 이에 해당한다. 높은 수준(high) 은 학습자가 학습 내용에서 발생하는 현상 의 이유를 궁금해하고, 어떻게 새로운 지식 및 다른 분야와 연결되는지 의문을 갖는 단계를 의미한다. 강의에서 다루었 던 내용이지만, 주어지지 않았던 설명을 요구하거나 ‘왜?' 라는 의문을 갖는 경우를 가장 높은 수준으로 정의하였다 [18]. 이는 관련 개념 등을 이해하지 못한 상태에서는 제 기하기 어려운 질문이며, 학습자가 학습 내용을 이해한 후 '만약' 등의 단어를 사용하여 학습 내용을 응용하며 제기할 수 있는 질문이다.

\section{2) 질문 주제 분석}

Harper et al.은 물리학 질문을 그 주제에 따라 식, 개념, 적용, 지식, 실험, 한계 총 6 가지로 범주화 및 정의하였다 [28]. 분석 항목에 부합하는 핵심어는 Table 2 에 나타내었 다. 식 (equation)은 학습자가 학습을 하는 데 있어서 식을 직접적으로 사용하거나 그 의미를 파악하는 것과 관련된 질문을 의미한다 [30]. 또한 식을 이해한다는 것은 학습자가 이를 통해 기초적인 개념을 파악하려 한다는 것으로 볼 수 있으며, 이는 수학적인 어려움과 깊은 관련이 있다. 그러므 로 핵심어 선정 시, '식'과 함께 등장하는 '수학'이라는 단어 
Table 3. Levels of question in physics (adapted from [18]).

\begin{tabular}{lll}
\hline \hline Level of question & Description & Keywords \\
\hline Minimal & $\begin{array}{l}\text { The question indicates that student has } \\
\text { a definitional problem. }\end{array}$ & introduce, define, what is, letter, unit, called, symbol \\
Lower & $\begin{array}{l}\text { The student has a problem understanding } \\
\text { the material presented by the instructor. }\end{array}$ & $\begin{array}{l}\text { understand, figure out, propose, mean, don't get, law, } \\
\text { what difference, can explain }\end{array}$ \\
& $\begin{array}{l}\text { The student has a procedural difficulty into } \\
\text { Medium }\end{array}$ & $\begin{array}{l}\text { confused, problem, difficulty, change, when can, during, } \\
\text { math, affect, correct, how come, come from }\end{array}$ \\
& $\begin{array}{l}\text { The student wants to know why something } \\
\text { happens or how new knowledge is connected } \\
\text { to other area. }\end{array}$ & $\begin{array}{l}\text { why, happen, connect, apply, what if, what would, relate, } \\
\text { wondering, assuming, if so, explain, proved, replace }\end{array}$ \\
\hline \hline
\end{tabular}

Note: Keywords were selected by the researchers of the present study.

를 포함시켰다. 개념 (concept)은 학습자가 학습의 주제나 의미에 의문을 갖거나 조금 더 나아가서 다른 영역으로 확 장하려는 의도를 가진 질문이다. 과학 개념에 대한 이해는 지식에 대한 확신의 정도와 유의미한 관계가 있어, 개념 을 질문한다는 것은 다른 영역으로의 지식 확장의 기초가 될 수 있다. 또한 학습자는 교수자의 특별한 지도 없이도, 자기주도적 학습을 통해 학습 내용과 관련된 특정 현상의 주의 깊은 관찰과 의문을 가지며, 이를 통해 물리학 개념의 이해를 구조화할 수 있다 [18].

적용 (application) 은 학습자가 학습한 내용을 일상경험 이나 다른 물리학 개념에 연관시키는 질문일 경우를 말한 다. 적용은 학습자들이 현실에서 교수내용을 적용시키면서 발생한 모순이나, 인지갈등 등을 유발시키는 부분이 되므 로, 질문을 통해 교수자 또는 다른 학습자와의 상호작용이 적극적으로 유발되는 부분이라고도 볼 수 있다 [24]. 지식 (knowing) 은 과학자들에 의해 개념화 또는 명명된 법칙이 학습자가 가지고 있는 지식과 어떠한 연관이 있는지 의문을 가질 때 하는 질문이다. 학습자가 학습을 하면서 이미 확립 된 여러 법칙이나 이론들의 개념과 차이점 등을 이해하고, 관점을 제대로 확립하는 것은 과학의 본성을 이해하는 데 핵심적인 영역이기도 하다 [31]. 실험 (experiment) 범주는 수업에 제시되거나 연관된 과학 실험에 있어서, 그것의 목적 이나 과정의 이해에 어려움을 가질 때 하는 질문이다. 전통 적인 교실 수업과 달리 $\mathrm{MOOC}$ 환경에서 실험은 학생이 직 접 수행하기보다는 간접적으로 경험할 수 있기 때문에 이에 대한 질문은 비교적 다양할 수 있다. 또한 간접 경험을 통해 관찰한 실험과 관련한 질문들은 학습자들이 학습 도중에 느끼는 인지갈등 및 구체적 현상에 대한 관찰 사실을 주로 나타낼 수 있다. 한계 (limitation) 는 물리학 개념, 법칙, 상 수 등의 적용범위에 대한 질문이다. 예를 들면, 중력가속도 $\mathrm{g}$ 값을 $9.8 \mathrm{~m} / \mathrm{s}^{2}$ 로 간주할 수 있는 경우가 어디까지인지에 대한 질문이 이에 해당한다.

3) 언어 네트워크 분석

언어 네트워크 분석(semantic network analysis) 은 특정 텍스트 집단 내에서 수많은 개념들이 의미론적으로 어떻게 연결되어 있는지를 조사하기 위해 사용되는 분석 방법이다. 언어 네트워크의 구성단위는 ‘단어'로, 이들 간의 연결성은 데이터 전체가 가진 의미 구조를 드러내는 데 사용될 수 있다 [31]. 또한 언어 네트워크 분석을 통해 특정 단어를 중점으로 하여 배열된 단어들의 구조적 특징을 분석함으 로써 텍스트의 의미를 심층적으로 이해할 수 있으며, 개념 사이의 관계를 파악할 수 있다. 특히 학습자의 질문 연구 에 활용되었을 때에는 학습자의 인지구조 내에 존재하는 의미를 도식적으로 재구조화하여 보여줄 수 있다는 점에서 유용하다 [31]. 본 연구에서 연구자는 네트워크 분석을 위해 NetMiner 4.5 (http://www.netminer.com) 를 사용하였으 며, 동의어, 제외어 등을 임의의 파일로 형성하여 지정하고 지정된 범위의 질문에서 나타나는 단어들을 분석하였다. 이 분석 방법에서는 매개성 높은 개념어들을 중심으로 네트 워크가 형성되며, 이를 통해 전체 개념들의 구조가 어떻게 나타나는지 알 수 있다. 또한 질문을 수준과 주제로 분류한 후 분석 결과들에 나타나는 개념구조들을 분석함으로써 학 습자 질문의 특징을 맥락적으로 도출할 수 있다.

\section{IV. 연구 결과}


Table 4. Topics of question in physics (adapted from [17]).

\begin{tabular}{|c|c|c|}
\hline Topic of question & Description & Keywords* \\
\hline Equation & $\begin{array}{l}\text { The student is concerned with the meaning of } \\
\text { or use of an equation. }\end{array}$ & equation, equal, change, formula, math \\
\hline Concept & $\begin{array}{l}\text { The student is concerned with the meaning of } \\
\text { a concept or the extension of the concept into } \\
\text { a different area and tries to make sense out of it. }\end{array}$ & $\begin{array}{l}\text { what, mean, define, solve, expand, (don't) understand, } \\
\text { comprehension, move, state, come from }\end{array}$ \\
\hline Application & $\begin{array}{l}\text { The student tries to relate the concept to } \\
\text { experience or other physics concepts. }\end{array}$ & $\begin{array}{l}\text { relate, apply, example, concerned, imagine, suppose, } \\
\text { same }\end{array}$ \\
\hline Knowing & $\begin{array}{l}\text { The student is interested in how a particular } \\
\text { knowledge was constructed by scientists. }\end{array}$ & how, interest, know, find out, figure out, discover \\
\hline Experiment & $\begin{array}{l}\text { The students don't understand the technical } \\
\text { details or the explanation of experiment. }\end{array}$ & experiment, purpose, process, result, case \\
\hline Limitation & $\begin{array}{l}\text { The students is interested in the range of } \\
\text { applicability of a concept, constant, etc. }\end{array}$ & range, applicable, extent \\
\hline
\end{tabular}

* Keywords were selected by the researchers of the present study.

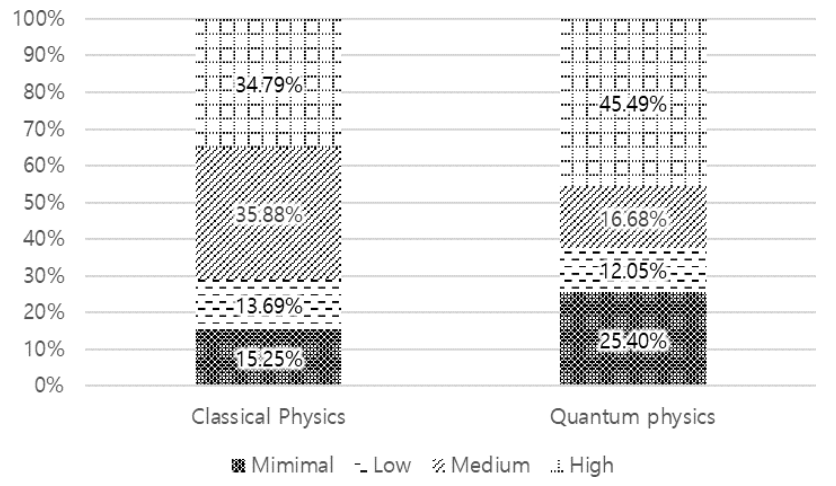

Fig. 1. Levels of questions in quantum and classical physics.

\section{1. 질문 수준}

고전물리학에 대한 어려움은 대부분 기초적인 개념에 관 한 것이 많다는 선행 연구와는 대조적으로 [32], 중간 수준 $(35.88 \%)$ 과 높은 수준 $(34.79 \%)$ 의 질문이 비슷한 양상으로 높게 나왔으며, 최소 수준의 질문 $(15.25 \%)$ 과 낮은 수준의 질문 $(13.69 \%)$ 은 비슷하게 낮은 빈도를 보였다(Fig. 1). 한 편 양자물리학 단원에서는 높은 수준의 질문 $(45.49 \%)$ 이 가장 많았으며, 두 번째로 최소 수준의 질문 $(25.40 \%)$ 이 많 은 것으로 나타났다. 이러한 결과는 거시적으로 볼 때 고 전물리학에서보다 양자물리학에서 질문 수준의 양극화가 심함을 시사하는데, 이 점은 이하에서 논의하였다.
I D

I dont understand how change of direction can effect acceleration? Formula $\mathrm{a}=\mathrm{v} / \mathrm{t}$ does not indicate that?

Reply - comments

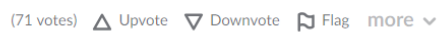

Fig. 2. (Color online) An example of medium-level questions in classical physics.

\section{1) 고전물리학 단원의 질문 수준}

고전물리학 단원에서 가장 높은 빈도수를 차지한 질문 은 중간 수준의 질문이었다. 중간 수준의 질문은 학습자가 학습을 이해하는 데 있어서 과정적인 어려움을 가진 것을 의미하므로, 학생들이 고전물리학의 원리 및 정의와 관련된 내용을 주로 질문하는 것으로 해석할 수 있다 [30].

고전물리학 단원의 중간 수준 질문의 예는 Fig. 2 와 같다. "방향의 변경이 가속도에 어떻게 영향을 미치는지가 이해 되지 않습니다. $a=v / t$ 공식에는 나타나지 않는데요?" 라는 이 질문에서 질문자는 강의에서 설명한 가속도 개념을 단편적으로만 이해하고 있으며 가속도가 크기와 방향을 포 함하는 벡터임을 이해하지 못하고 있다. 따라서 이 질문을 제기한 학습자는 교수자에 의해 제시된 강의자료의 의미를 1 차적으로는 이해하고 있으나, 여기에서 나아가 물리량과 물리공식 사이의 관계를 이해하는 데 어려움을 표현한 것 으로 보아 과정적인 어려움을 갖고 있다고 해석할 수 있다. 


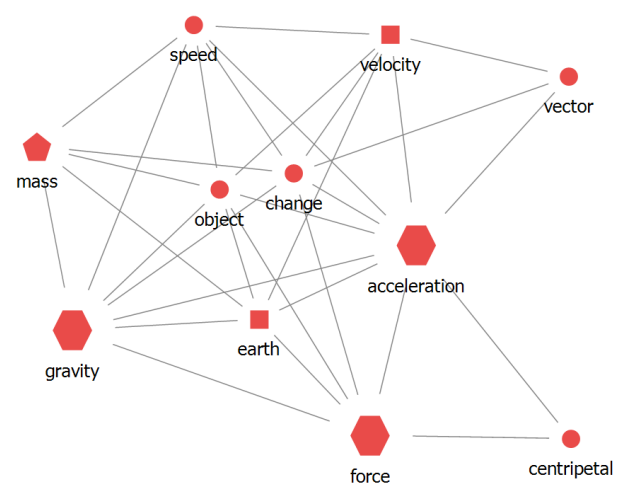

Fig. 3. (Color online) Semantic network analysis of medium-level questions in classical physics.

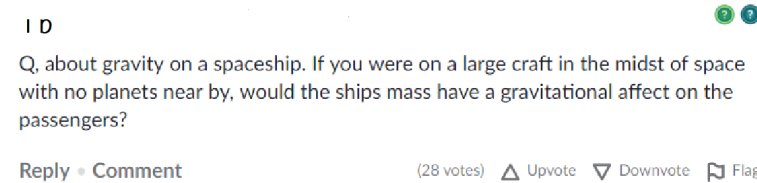

Fig. 4. (Color online) An example of high-level questions in classical physics.

고전물리학 단원에서 중간 수준의 질문이 가장 높은 비율 로 나타났기 때문에, 중간 수준 질문에 대하여 심층적으로 알아볼 필요가 있다. 이를 알아보기 위하여 실시한 언어 네트워크 분석 결과는 Fig. 3 과 같다. 고전물리학 중간 수 준의 질문은 빈도수가 가장 높은 힘 (force) 개념에서 시작하 여 중력 (gravity), 가속도(acceleration)와 삼각형을 이루며 가장 많이 연관되어 있다. 이를 통해 '힘이 중력가속도와 어떠한 연관을 가지고 있는지'가 중간 수준 질문의 가장 핵심적인 아이디어임을 추론할 수 있다. 또한 기타 주요 단 어들을 통해 중간 수준의 질문들은 지구를 중심으로 질량, 속도, 속력, 가속도, 힘, 중력이 어떻게 서로 연관되는지를 중심으로 제기되고 있음을 알 수 있다.

고전물리학 단원에서 두 번째로 높은 빈도수를 차지한 질문은 높은 수준의 질문이었다. 높은 수준의 질문은 학 습자가 강의를 듣고 제기한 질문 중 탐구적인 내용이 가장 많았으며, 이는 과학적 흥미와 연관되어 점차 높은 수준의 과학적 주제로 학습을 이끌어 가는 동기가 된다.

고전물리학 단원의 높은 수준 질문의 예는 Fig. 4 와 같다. “우주선의 중력에 대한 질문으로, 근처에 행성이 없는 우주 의 한 가운데 있는 큰 우주선에 당신이 타고 있다면, 우주선 질량이 승객들에게 중력 효과를 가질까요?"라는 해석에서 볼 수 있듯이 이 질문을 제기한 학습자는 중력이라는 개념을 지구의 질량에 의한 중력에 한정하여 이해하지 않고, 우주 공간에서 우주선 자체의 질량에 의해 발생하는 중력 효과를
I D

So if a neutron becomes a proton by expelling an electron, and a proton becomes a neutron by expelling a positron, and an electron has the same mass as a positron, then why is there a slight discrepancy in mass between a neutron and a proton?

Reply c comment $\quad$ (25 votes) $\Delta$ Upvote $\nabla$ Downvote $\quad \boldsymbol{a}$ Fläg

Fig. 5. (Color online) An example of high-level questions in quantum physics.

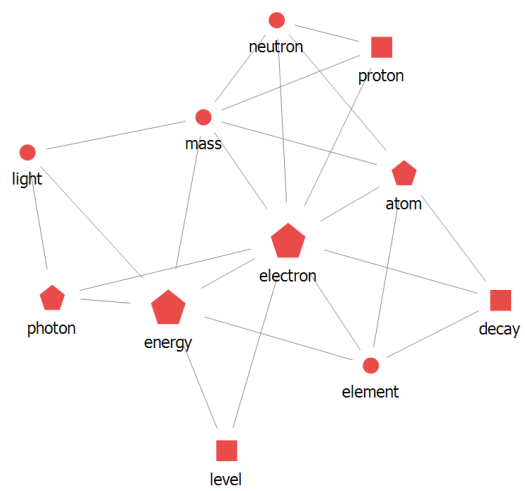

Fig. 6. (Color online) Semantic network analysis of highlevel questions in quantum physics.

고려할 수 있는지를 질문하고 있다. 이 질문의 예시에서 확 인할 수 있듯이 학습자는 높은 수준의 질문을 통해 스스로 연구 질문을 이끌어내고 있다고 짐작할 수 있다.

\section{2) 양자물리학 단원의 질문 수준}

양자물리학 단원에서는 높은 수준의 질문이 $45.49 \%$ 로 가장 많이 나타났다. 그러나 이 결과를 학습자들이 양자물 리학의 문제들에 대해 물리학적으로 정교하고 심오한 질 문을 던지고 있다고 해석하기보다는, 양자물리학 단원의 특성상 파동성과 입자성, 미시세계와 거시세계 등 상반되는 개념들이 복합적으로 관여하며 또한 반직관적인 현상이나 설명들이 다수 존재한다는 점에서 원인을 찾는 것이 타당할 것이다. 높은 수준의 질문은 과학을 학습하면서 왜 그러한 현상이 발생하는지, 다른 물리학적 현상과 어떻게 연결되 는지를 이해하고자 제기하는 질문이므로, 이와 같은 양자 현상의 특징이 양자물리학을 수강한 학습자로 하여금 다양 한 종류의 높은 수준 질문을 유발하였다고 볼 수 있다.

높은 수준 질문의 예는 Fig. 5 와 같다. "만약 중성자가 전자를 방출함으로써 양성자가 되고, 양성자는 양전자를 방출함으로써 중성자가 되고, 전자는 양전자와 같은 질량을 가진다면, 왜 중성자와 양성자 간의 질량에 약간의 불일치가 
I D

What are photons?

Reply * Comment

I D

It is easiest to say that it is a particle that is light. basically, it has no mass, and therefore can reach light speeds.

1 comment

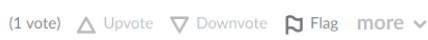

Fig. 7. (Color online) An example of low-level questions in quantum physics.

있을까요?"라는 질문은 수업에서 명시적으로 언급된 적이 없지만 기본 입자 사이의 질량관계라는 점에 관해 새로운 의문을 제기하고 있다는 점에서 창의적인 질문으로 볼 수 있다. 양자물리학 단원에서 제기된 높은 수준 질문들의 개 념적 연관 관계에 대해 심층적으로 알아보기 위하여 실시한 언어 네트워크 분석 결과는 Fig. 6 과 같다.

양자물리학 단원의 높은 수준 질문은 에너지 (energy)와 전자(electron) 라는 단어를 축으로 네트워크가 형성되어, 전자의 에너지에 관한 질문이 핵심이 되고 있음을 알 수 있 다. 우선, 전자(electron) 를 중심으로 질문의 연결 흐름을 보면, 양성자(proton), 중성자(neutron) 및 전자(electron) 로 구성된 원자(atom)에 대한 질문이 있고, 전자(electron) 와 원자(atom)축을 공통으로 전자 에너지 준위에 대한 내 용으로 이어짐을 알 수 있다. 양자물리학 단원의 높은 수준 질문을 분석한 결과 대부분 물질의 구조와 관련된 단어가 연관되어 있음을 것을 알 수 있으며, 따라서 기본 입자 사 이의 관계성을 통해 물질의 미시적 구조를 이해하는 것이 양자물리학 단원을 관통하는 핵심 과제가 되고 있음을 추 론할 수 있다.

높은 수준의 질문 다음으로는 최소 수준의 질문이 $25.40 \%$ 로 두번째로 높았다. 이는 학습자의 양자물리학 이해에 있 어서 양극화가 심하다는 것을 의미한다. 최소 수준의 질문 이 비교적 많이 제기됐다는 것은 양자물리학의 단원 특성상 학습자들이 해당 단원 학습을 중도 포기하는 경우가 많음 을 짐작할 수 있다. 이에 반해 양자물리학 단원에서 높은 수준의 질문이 많았다는 것으로 보아 물리학에 관심과 흥 미가 높은 학습자들은 양자물리학 단원에서도 탐구적이고 도전적인 질문들을 제기한다는 것을 짐작할 수 있다. 또한 양자물리학 단원에서 중요하게 다루는 개념에 대한 이해가 많이 부족하기 때문에 최소 수준의 질문 빈도가 높았다는 것을 확인할 수 있다.

양자물리학 단원의 최소 수준 질문의 예는 Fig. 7 의 "광자 가 무엇이죠?”와 같은 질문에서 확인할 수 있다. 이 질문이 제기된 강의는 '광자 에너지' (photon energy) 차시로서, 총 강의 시간 9 분 17 초 중 약 1 분경부터 2 분의 시간동안 강사

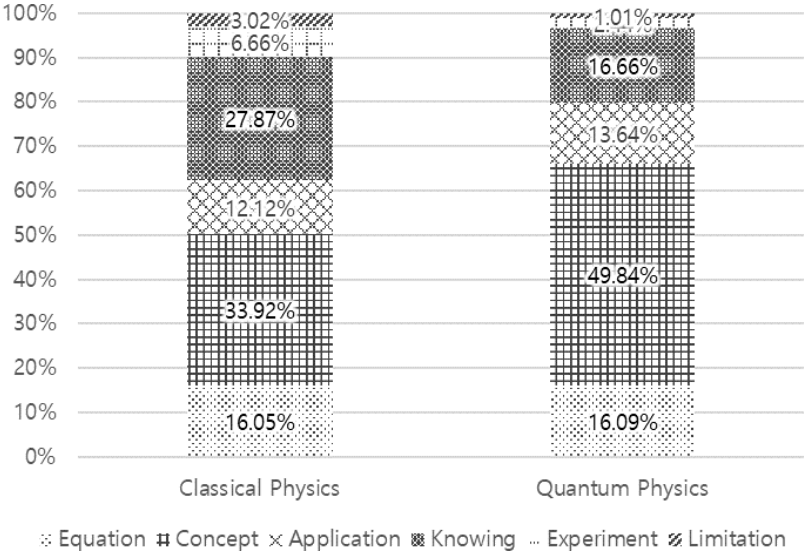

Fig. 8. Topics of questions in quantum and classical physics.

가 양자 도약, 빛의 기본 입자, 광자의 개념, 광자의 성질 등 광자에 관한 내용을 자세히 설명함에도 불구하고 학생은 광자에 관한 포괄적인 질문을 제기하고 있다는 점에서, 이 질문은 해당 강의를 전반적으로 이해하지 못한 상태에서 제기한 최소 수준의 질문이라고 볼 수 있다. 이 질문에 대한 답변을 단 사용자 역시도 광자에 대한 원론적이고 교과서 적인 설명만을 반복하고 있는 것을 볼 수 있다(Fig. 7).

\section{2. 질문 주제}

고전물리학 단원의 질문을 주제로 분석한 결과는 Fig. 8 과 같다. 개념 (concept) 질문이 $33.92 \%$ 로 가장 높았으며, 지식(knowing) 질문이 $27.87 \%$, 식 관련 질문이 $16.06 \%$, 적 용 질문이 $12.12 \%$ 으로 뒤를 이었으며, 실험 질문이 $6.66 \%$, 한계 질문이 $3.02 \%$ 로 낮은 양상을 보였다(Fig. 8). 양자 물리학 단원의 질문을 주제로 분석한 결과도 고전물리학 단원과 마찬가지로 개념 질문 $(49.84 \%)$ 이 가장 많았다. 다 음으로 지식 질문이 $16.66 \%$, 식 질문이 $16.09 \%$ 로 비슷하게 나타났으며, 적용 질문이 $13.64 \%$, 실험 질문이 $2.44 \%$, 한계 질문이 $1.01 \%$ 로 미비하게 나타났다. 고전물리학 단원과 양자물리학 단원에서 질문의 주제에 따른 분류 결과는 비슷 한 양상을 보였으며 두 단원 모두 개념과 지식 관련 질문이 많았다.

\section{1) 고전물리학 단원의 질문 주제}

고전물리학 단원에서 질문을 주제별로 분석한 결과, 개념 질문이 $33.92 \%$ 으로 가장 많이 나타났다. 개념 질문은 학습 자가 학습의 주제나 의미에 의문을 갖는 질문으로 학습의 
I D

what is centrifugal force? and does it exist or not?

Reply * Comment (1 vote) $\Delta$ Upvote $\nabla$ Downvote $\boldsymbol{\nabla}$ Flag more $\checkmark$

4) 1 D

what is the magnitude?

Reply * Comment

Fig. 9. (Color online) An examples of “concept" questions in classical physics.

I D

What exactly do we mean by 'energy levels' ?

A hydrogen atom is in possession of a single 1s2 orbital, wherein there exists a single electron. If $i$ were to provide this electron with a certain amount of energy, as i understand it, it would move to a higher energy level, but doesn't a hydrogen atom contain only a single orbital? where does this electron actually go? does it still remain within the orbital?

Reply - Comment

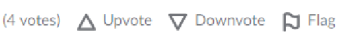

Fig. 10. (Color online) An example of “concept" questions in quantum physics.

이해 정도와 깊은 관련이 있다. 이외에 지식 $(27.87 \%)$, 식 $(16.05 \%)$, 적용 $(12.12 \%)$, 실험 $(6.66 \%)$, 한계 $(3.02 \%)$ 순서 로 질문의 주제가 분포하였다.

고전물리학 단원의 개념 질문의 예는 Fig. 9 와 같다. "원 심력은 무엇이고, 그것은 존재하나요 아니면 존재하지 않나 요?” 라는 질문은 원심력에 대한 개념을 이해하지 못하기 때문에 제기된 질문이라 볼 수 있다. 원심력은 구심력과 크기는 같고 반대 방향이며, 일정한 각속도로 회전하는 관 성력이다. 위 질문이 제기된 강의에서도 예시로 커브길을 도는 자동차를 설명하였는데, 여기에서 실제로 커브길을 도는 자동차 안에 있는 사람은 커브 바깥쪽으로 쏠리는 힘 을 받는다. 강의에서도 이 현상을 관성에 의한 효과일 뿐 실제로 존재하지 않는다고 설명된 바 있다.

\section{2) 양자물리학 단원의 질문 주제}

양자물리학 단원에서는 개념 질문이 $49.84 \%$ 으로 가장 많 이 나타났다. 이러한 분석 결과는 고전물리학 단원에서도 동일하게 도출되었으므로, 칸 아카데미의 두 단원 모두에서 학습자들은 개념 질문을 가장 많이 제기하고 있음을 확인할 수 있다. 이외에 지식 $(16.66 \%)$, 식(16.09\%), 적용(13.64), 실험 $(2.44 \%)$, 한계 $(1.01 \%)$ 순으로 질문이 분포하였다.

양자물리학 단원의 개념 질문의 예는 Fig. 10 와 같다. "에너지 수준이란 정확히 무엇을 의미하나요? 수소 원자는 단일 전자가 존재하는 단일 $1 \mathrm{~s} 2$ 궤도를 가지고 있습니다.

만약 이 전자에 일정량의 에너지를 공급한다면, 이것은 더 높은 에너지 준위로 이동하지만, 수소 원자는 단 하나의 궤도만을 포함하지 않나요? 이 전자는 실제로 어디에 있나 요? 여전히 궤도 안에 남아 있나요?" 라는 질문은 개념 확 인 질문으로 볼 수 있다. 이를 통해 학습자는 에너지 준위에 대해 이해는 했지만, 빛의 흡수나 들뜸 현상 같은 그 다음 개념으로 생각을 이어가지 못하고 있음을 확인할 수 있다.

\section{3. 질문 수준과 질문 주제의 상관관계}

고전물리학 단원에서 질문의 수준과 질문 주제의 상관 관계를 살펴보면(Table. 5) (1) 최소 수준에서 개념 질문 이 $74.7 \%$, 낮은 수준의 질문에서도 개념과 관련된 질문 이 $47.2 \%$ 로 가장 많았고, (2) 중간 수준에서는 지식 관련 질문이 $31.1 \%$, 높은 수준의 질문에서는 지식 관련 질문이 $37.5 \%$ 로 가장 많았다. 한편 고전물리학 단원에서는 최소 수준부터 높은 수준까지 개념 질문이 높은 비율을 차지하는 것으로 나타나 학습자들이 다양한 수준의 개념 관련 질문을 제기하고 있음을 알 수 있다. 지식 관련 질문 역시 낮은 수준부터 높은 수준에 걸쳐 점점 그 비율이 증가하는 양상 을 보여 학생들이 고전물리학 지식의 형성 배경, 또는 특정 물리학 내용에 대해 '우리가 그것을 어떻게 아는지'에 대한 질문을 빈번하게 제기하고 있으며, 이러한 질문의 비율은 질문 수준이 높아지면서 점점 늘어남을 알 수 있다.

양자물리학 단원에서는 (1) 높은 수준의 질문이 가장 많 이 발견되었으며, (2) 최소 수준에서 개념질문은 $64.7 \%$, 낮은 수준에서는 $64.2 \%$, 중간 수준에서는 $45.6 \%$, 높은 수 준에서는 $39.3 \%$ 로 모든 수준의 질문에서 개념 질문이 가장 많으면서도 그 비율이 질문 수준이 높아지면서 점차적으로 감소하는 경향을 보였다. 또한 질문 수준이 높아짐에 따라 양자물리학의 중요 개념인 광자, 원자의 구조, 핵붕괴와 핵 분열, 핵융합 등을 기존의 일상 지식이나 기타 물리학 지식 과 연관짓는 적용 관련 질문의 비율이 전반적으로 증가하는 경향을 보였다.

\section{V. 결론 및 시사점}

본 연구는 $\mathrm{MOOC}$ 상에 나타난 '구심력과 중력' 단원 질 문과 양자물리학 질문을 질문의 수준과 주제를 기준으로 분석하고 언어 네트워크 분석을 통해 일부 범주의 질문을 심층적으로 분석하였다. 연구를 통하여 도출한 $\mathrm{MOOC}$ 상의 질문 분석 결과는 다음과 같은 물리교육적 활용가능성 및 시사점을 갖는다. 첫째, $\mathrm{MOOC}$ 를 통해 나타나는 질문들을 
Table 5. Questions in Khan Academy by question levels and question topics.

\begin{tabular}{lllll}
\hline \hline & Minimal & Lower & Medium & High \\
\hline & Equation: $11.2 \%$ & Equation: $10.9 \%$ & Equation: $23.8 \%$ & Equation: $12.3 \%$ \\
& Concept: $74.7 \%$ & Concept: $47.2 \%$ & Concept: $19.9 \%$ & Concept: $25.1 \%$ \\
Classical & Application: $2.1 \%$ & Application: $13.6 \%$ & Application: $18.8 \%$ & Application: $9.2 \%$ \\
physics & Knowing: $8.2 \%$ & Knowing: $17.6 \%$ & Knowing: $31.1 \%$ & Knowing: $37.5 \%$ \\
& Experiment: $2.8 \%$ & Experiment: $7.0 \%$ & Experiment: $3.0 \%$ & Experiment: $12.1 \%$ \\
& Limitation: $1.0 \%$ & Limitation: $3.7 \%$ & Limitation: $3.4 \%$ & Limitation: $3.8 \%$ \\
& Equation: $11.2 \%$ & Equation: $13.7 \%$ & Equation: $21.3 \%$ & Equation: $17.5 \%$ \\
& Concept: $64.7 \%$ & Concept: $64.2 \%$ & Concept: $45.6 \%$ & Concept: $39.3 \%$ \\
Quantum & Application: $4.7 \%$ & Application: $2.4 \%$ & Application: $7.2 \%$ & Application: $24.2 \%$ \\
& Knowing: $17.7 \%$ & Knowing: $17.3 \%$ & Knowing: $18.3 \%$ & Knowing: $15.3 \%$ \\
& Experiment: $1.0 \%$ & Experiment: $1.2 \%$ & Experiment: $6.2 \%$ & Experiment: $2.2 \%$ \\
& Limitation: $0.7 \%$ & Limitation: $1.2 \%$ & Limitation: $1.4 \%$ & Limitation: $1.3 \%$ \\
\hline \hline
\end{tabular}

질문의 수준 및 질문의 주제에 따라 비교 분석한 결과를 토대로 교수경험이 없는 예비교사들에게 물리학 개념학습 과정에서 학생들이 제기하는 질문들을 간접적으로 경험해 보도록 할 수 있다. 교실에서보다 방대하고 다양한 질문의 양상을 보이는 $\mathrm{MOOC}$ 강의의 특성상 학생 이해의 어려 움을 심층적으로 이해하는 데 도움이 될 것으로 기대된다. 둘째, $\mathrm{MOOC}$ 플랫폼상 질문의 언어 네트워크 분석을 통 하여 학생들이 어려워하는 개념 구조를 수준별, 주제별로 확인함으로써, 향후 온라인 환경에서 물리학 수준별 맞춤 학습전략을 제공하는 데 유용한 자료로 사용될 수 있다.

$\mathrm{MOOC}$ 환경에서의 물리학 질문에 대한 탐색적 연구로 서 본 연구의 한계점은 다음과 같다. 첫째, 칸 아카데미 물리학 강의의 내용과 국내 중등 물리학 교육과정의 내용 및 범위상에 차이가 있으므로 이를 분석한 결과를 국내 상 황에 곧바로 일반화하여 적용하기 어렵다는 제한점이 있다. 둘째, 물리학 학습자가 제기하는 질문은 구체적인 교육과 정의 구성뿐만 아니라 $\mathrm{MOOC}$ 강의별 교수자의 전달방식, 플랫폼별 기능의 차이 등에 의해 영향을 받을 수 있다. 이 를테면 원심력과 중력 단원에서 교수자가 일상경험과의 연 계를 강조하는 수업방식을 택할 경우 그와 연관된 질문의 빈도가 높아질 수 있다. 이와 같은 두 가지 한계점을 고려할 때, 향후 국내에서 중등학교 수준 $\mathrm{MOOC}$ 물리학 강의에 대한 추가적인 논의가 진행되기 위해서는 국내 교육과정과 환경에 맞추어진 플랫폼을 바탕으로 학습자의 질문을 적극 장려하고, 이를 분석하려는 시도가 필요하다고 할 것이다.

학생의 물리학 오개념에 대해 수십 년간 다수의 연구가 진행되어 왔으나, 학생이 적극적으로 제기한 질문을 물리학 단원별로 접근한 연구는 드물다. 이는 개별 교실 상황에서 다양한 유형, 수준, 주제의 학생 질문을 폭넓게 관찰하기 어렵다는 점에 기인한다고 볼 수 있다. 이러한 점에서 본
연구는 전세계의 다수 학습자들이 사용하는 $\mathrm{MOOC}$ 교육 플랫폼인 칸 아카데미에 등록된 방대한 양의 댓글들을 바탕 으로 특정 물리학 단원의 다양한 학생 질문을 분석함으로써 빅데이터에 기초한 물리학 학습 연구의 한 예를 제시하였 다는 점에 부가적인 의의가 있다. 향후 칸 아카데미와 유 사한 외국 및 국내 $\mathrm{MOOC}$ 플랫폼을 대상으로 한 지속적인 연구를 통해 국내 상황에 적합한 $\mathrm{MOOC}$ 물리학 환경 조성 및 물리학 학습 지원 방안을 도모할 필요가 있다.

\section{감사의 글}

본 논문은 2016년 정부(교육부)의 재원으로 한국 연구재단의 지원을 받아 수행된 연구입니다 (NRF2016S1A3A2925401).

\section{REFERENCES}

[1] NGSS Leads States, Next Generation Science Standards (National Academy Press, 2013).

[2] N. Hood, A. Littlejohn and C. Milligan, Comp. Edu. 91, 83 (2015).

[3] M. Gaebel, MOOCs - Massive Open Online Courses(EUA Occasional papers, 2013)

[4] S. Khan, The one world schoolhouse: Education reimagined(Twelve, 2012).

[5] Khan Academy Korea homepage, http://khanacademy koreancommunity.org (accessed Sep., 15, 2018). 
[6] Khan Academy Korea Teacher Guide homepage, https://www.edwith.org/khanacademy_guide (accessed Sep., 15, 2018).

[7] Y. W. Cheong, J. Song, New Phys: Sae Mulli 61, 713 (2011).

[8] Byun, T., Ph.D. thesis, Seoul National University, 2012

[9] S. Park, M.A. thesis, Seoul National University, 2014

[10] H. Jho, New Phys: Sae Mulli 68, 869 (2018).

[11] B. W. Lee, S. M. Lee, New Phys: Sae Mulli 49, 37 (2004)

[12] H. R. Sadaghiani, Ph.D. thesis, The Ohio State University, 2005

[13] G. Marbach-Ad, P. G. Sokolove, J. Res. Sci. Teach. 37, 854 (2000).

[14] C. Chin, D. E. Brown, Int. J. Sci. Edu. 24, 521 (2002).

[15] S. Cuccio-Schirripa, H. E. Steiner, J. Res. Sci. Teach. 37, 210 (2000).

[16] E. Buckner, P. Kim, Prospects 44, 99 (2014).

[17] S. Yerdelen-Damar, A. Eryılmaz, Res. Sci. Edu. 40, 223 (2010).

[18] E. Etkina, Sci. Edu. 84, 594 (2000).

[19] C. Chin and J. Osborne, Stud. Sci. Edu. 44, 1 (2008).
[20] A. Hofstein, O. Navon, M. Kipnis and R. Mamlok-Naaman, J. Res. Sci. Teach. 42, 791 (2005).

[21] Y. Ko, H. Lee, J. Korean Assoc. Sci. Edu. 34, 449 (2014)

[22] Ministry of Education, The Science Curriculum, (2015)

[23] A. Baram-Tsabari, R. J. Sethi, L. Bry and A. Yarden, Sci. Edu. 90, 1050 (2006).

[24] S. G. Kim, S. I. Yeo and K. W. Woo, J. Korean Assoc. Sci. Edu. 19, 377 (1999)

[25] H. A. Orr, Science 285, 343 (1999).

[26] Y. W. Cheong, J. Song, New Phys: Sae Mulli 61, 713 (2011)

[27] Y. Ko, H. Lee and S-. W. Kim, New Phys: Sae Mulli 65, 812 (2015).

[28] K. A. Harper, E. Etkina. and Y. Lin, J. Res. Sci. Teach. 40, 776 (2003).

[29] E. Kaya, S. Erduran, Sci. \& Edu. 25, 1115 (2016).

[30] M. Kim, Ph.D. thesis, Seoul National University, 2016

[31] J. K. Lee and M. Ha, J. Korean Assoc. Sci. Edu. 32, 823 (2012)

[32] D. S. Kim and E. A. Cho, Bull. Sci. Edu. 13, 59 (1997) 